CP, 2018, Vol.7 - No15, pp. 231/245 ISSN 2014-6752. Girona (Catalunya). Universitat de Girona. LÓPEZ DE AYALA, María Cruz; CATALINA Beatriz; FERNÁNDEZ, José Gabriel: Estrategias de las empresas de campos de golf en Twitter. Su influencia sobre la interactividad de los públicos Recibido: 11/05/2018 - Aceptado: 23/10/2018

\title{
Estrategias de las empresas de campos de golf en Twitter. Su influencia sobre la interactividad de los públicos
}

\section{Strategies of golf course companies on Twitter. Its influence on the interactivity of the public}

\author{
AUTORES: \\ María Cruz López de Ayala \\ http://orcid.org/0000-0002-6989-866X \\ Universidad Rey Juan Carlos. Profesor Titular. Departamento de Comunicación y Sociología \\ Beatriz Catalina \\ http://orcid.org/0000-0003-0464-3225 \\ Universidad Rey Juan Carlos. Profesor visitante. Departamento de Comunicación y Sociología \\ José Gabriel Fernández \\ http://orcid.org/0000-0001-6860-2989 \\ Universidad Rey Juan Carlos. Profesor titular. Departamento de Comunicación y Sociología
}

\section{Resumen}

Diversas investigaciones han constatado la relación entre la interactividad en redes sociales y la actitud de los usuarios hacia las empresas, generando interés por conocer qué herramientas y tácticas pueden fomentar la actividad de los seguidores en los medios sociales. Mediante un seguimiento y análisis de contenido de los perfiles activos en Twitter de los clubs con campos de golf en la Comunidad de Madrid a lo largo de 2016, se analiza la influencia de las temáticas de los tuits publicados, la inclusión de recursos audiovisuales y de mensajes retuiteados sobre el grado de difusión, selección como favoritos y la inclusión de comentarios por parte de los seguidores. Entre las principales conclusiones destaca la efectividad de los mensajes educativos, de estilo de vida, saludos y concursos, preguntas y sorteos, muchos de ellos retuiteados, para favorecer la interactividad; por el contrario, se observa un impacto positivo limitado del uso de recursos multimedia sobre la respuesta de los usuarios. En defi-

\section{Abstract}

Several studies have confirmed the relationship between interactivity in social networks and the attitude of users towards companies, raising interest to know the effectiveness of tools and tactics to promote the participation of their audiences. Through monitoring and a content analysis of the active profiles that golf clubs of the Community of Madrid have on Twitter during 2016, the effects of the topics of the tweets published in these accounts, the inclusion of audio-visual resources and retweeted messages on the diffusion, selection as favourites and the inclusion of comments are examined. Key findings include the effectiveness of educational messages, lifestyle, greetings and contests, questions and raffles, many of them retweeted, in order to promote interactivity; on the contrary, there is a limited positive impact of the use of multimedia resources on the response of users. In short, the inclusion of competitions, questions or raffles is valued as a convenient formula to get audience participation, but an adequate 
nitiva, la inclusión de concursos, preguntas o sorteos se valora como una fórmula conveniente para conseguir la participación de la audiencia, pero se precisa una selección adecuada de los mismos. Asimismo, se recomienda compartir contenidos de otros perfiles y saludos, e incluir videos.

Palabras clave: clubs de golf; comunicación corporativa; efectividad; interactividad; redes sociales. selection of them is needed. It is also recommended to share content from other profiles and greetings, and include videos.
Keywords: golf course; Corporate communication; effectiveness; interactivity; social networks

\section{Introducción}

Desde ámbitos académicos y profesionales, se ha destacado el papel que los medios sociales interactivos pueden cumplir en la construcción de relaciones entre las organizaciones y sus públicos estratégicos (Cornelissen, 2008), favoreciendo el diálogo y mejorando el compromiso entre ambos (Bowen, 2013; Grunig, 2011; Kang, 2014; Vernuccio, 2014; Watkins, 2017). En coherencia con esta idea, los expertos coinciden en afirmar que los medios sociales están revolucionado las prácticas comunicativas de las organizaciones con sus públicos estratégicos (Sung y Kim, 2014; Wright y Hinson, 2015).

En la misma línea, los resultados del estudio Latinoamerican Communication Monitor (Moreno, Molleda, Athaydes y Suárez, 2017) ponen de manifiesto que el 83\% de los profesionales de la comunicación consideran a las redes sociales como el canal más importante para la gestión de la comunicación, y a la revolución digital y la web social como el principal reto al que debe hacer frente la disciplina, preocupación que ha ido escalando puntos en los últimos diez años.

El interés por las potencialidades de los medios para mejorar el compromiso de los públicos con las empresas ha generado un creciente cuerpo de investigación que analiza la presencia y el uso de medios sociales entre las compañías más activas o reputadas y en distintos sectores. Estos estudios constatan la relación entre la interactividad en las cuentas y la actitud y compromiso de los seguidores con las empresas titulares de las cuentas. Sin embargo, existe una carencia de trabajos científicos que analicen la efectividad de distintas herramientas y tácticas para fomentar la interactividad de los públicos como base para mejorar la imagen y la lealtad hacia las organizaciones.

El objetivo de este artículo es conocer la efectividad de las medidas que los clubs con campos de golf están implementando en su gestión en Twitter para fomentar la comunicación bidireccional con sus públicos, y se examina la respuesta que el tipo de mensaje genera entre los usuarios. Asimismo, se analiza el grado de interactividad alcanzado por la inclusión de recursos audiovisuales o el uso de retuits en la gestión de esta plataforma.

\subsection{Prácticas en redes sociales y participación de los públicos}

Los medios sociales interactivos han ido adquiriendo una importancia creciente en la percepción que los públicos se forman de una empresa (Dijkmans, Kerkhof, Buyukcan-Tetik y Beukeboom, 2015). Un aspecto esencial de la gestión en redes sociales que se relaciona con 
la actitud de los públicos hacia la organización es su interactividad y compromiso de participar activamente en estos espacios (Kim, Sung y Kang, 2014; Li y Li, 2014; Men y Tsai, 2015 y 2013). Recientemente, desde Interactive Advertising Bureau (IAB, 2017) también se ha subrayado que existe una relación entre las marcas que logran una mayor comunidad y las interacciones que obtienen.

A pesar de las expectativas creadas, el principal motivo de los usuarios para seguir a las marcas en redes sociales no es interactuar con ellas: del $83 \%$ de los internautas que dicen seguir a las empresas en las redes sociales, solo un $6 \%$ lo hizo por esta razón. Por el contrario, los motivos principales que manifiestan los usuarios españoles para empezar a seguir a las marcas en redes sociales son: para mantenerse informado (47\%) y conocer sus productos/servicios (36\%). El $35 \%$ comienzan a seguir a las marcas porque es la que les gusta, en tanto que el $36 \%$ lo hacen para participar en un concurso (27\%) u obtener descuentos de la marca (24\%). Por otra parte, la razón más mencionada para continuar siendo fan de las marcas en redes sociales es que publiquen contenidos interesantes (42\%), actuales (39\%) y promociones (34\%). Sin embargo, se destaca la caída en la fidelización por ofertas (IAB, 2016b).

Numerosos expertos han aportado diferentes propuestas, basadas en el diálogo y la participación activa de los usuarios, para que las organizaciones puedan extraer el máximo provecho de su presencia en redes sociales. Entre otros aspectos, se apunta a un uso de los medios sociales que sea mutuamente beneficioso tanto para la entidad como para su público (Voss y Kumar, 2013), y la publicación de contenido interesante y relevante para estos últimos (Edman, 2010; Hallahan, 2006).

Sung y Kim (2014) trabajan sobre la hipótesis de una mayor efectividad de los mensajes no promocionales sobre los que sí lo son con el objetivo de mejorar la evaluación positiva de la empresa. Entienden por mensajes promocionales aquellos que, con fines comerciales, se relacionan con la experiencia de una empresa en sus productos y servicios. Los mensajes no promocionales se enfocan en crear amistades con los usuarios a través de conversaciones casuales, e incluyen consejos útiles sobre la vida, temas de actualidad o saludos estacionales o diarios. Aunque los autores no encuentran esa relación, sugieren que una estrategia no-promocional y de alta interactividad puede ser útil en la búsqueda de nuevos públicos potenciales inactivos en la relación con las compañías en medios sociales.

Por el contrario, algunos estudios muestran que, precisamente, esta actividad de marketing promocional excesiva de algunas compañías es la principal razón por la cual las personas dejan de seguirlas en Facebook (McCorkindale, 2010). Y Ruiz-Iniesta (2012) encuentra que los temas corporativos generan más quejas y reproches por parte de los usuarios. Mientras que Etter (2013) explica que los bajos niveles de interacción alcanzados por los mensajes de responsabilidad social corporativa se deben a su carácter reactivo y la carencia de especialización.

Apoyándose en datos cuantitativos y cualitativos, Sisson (2017) ofrece, entre otras, las siguientes recomendaciones orientadas a crear estrategias basadas en el diálogo y la escucha entre las partes: requerir comentarios de forma habitual; facilitar el diálogo para recoger opiniones valiosas, e implementar sugerencias regularmente. Y Men y Tsai (2012) concretan que las organizaciones pueden crear contenido de medios sociales que sea entretenido y útil con 
elementos multimedia, encuestas interactivas y juegos para aumentar el compromiso con los públicos. Otros autores que inciden en este aspecto son Herrera y Requejo (2012), quienes recuerdan que, según los especialistas en la materia, la interacción con la audiencia se puede favorecer haciendo retuits, realizando encuestas, difundiendo eventos en vivo o presentando pruebas cuando informan.

En el ámbito de la administración pública, Farranha y Dos Santos (2015), recomiendan la inclusión en los tweets de frases cortas y claras que lleguen a todos los perfiles y que se completen con recursos que llamen la atención, como fotografías, gráficos o hagstags para fomentar la participación. Otros autores que insisten en la incorporación de funciones multimedia para aumentar la frecuencia de "likes", comentarios y difusión son Gao (2016) y Kim, Kim y Sung (2011). Asimismo, Aced y Lalueza (2016) sugieren incluir más enlaces y vídeos para fomentar los "me gusta" e incrementar los contenidos que generan más actividad en los seguidores, como la información sobre la empresa y sus productos y servicios. Por último, IAB (2016a) también recomienda, además de utilizar influencers y celebrities adaptados a los valores de marca para incrementar la amplificación y la interacción con los usuarios, incluir formatos audiovisuales, contenido sencillo, emocional y cercano que transmita un estilo de vida además de aprovechar hitos y tendencias de actualidad.

\subsection{Twitter y el golf en la Comunidad de Madrid}

Según confirma el Libro Blanco de la Federación de Golf de Madrid (2013), el golf es el deporte con mayor número de federados en la Comunidad de Madrid. También es esta el área territorial autonómica con más aficionados a esta práctica en España y cuenta con 37 clubes, ya sea de 18 o de 9 hoyos (Federación de Golf de Madrid, 2017).

Aunque la industria del golf adquiere una gran importancia económica capaz de generar 2.000 millones de euros al año en España (Aymerich y Anabitarte, 2016), el sector lucha constantemente contra prejuicios acerca de la naturaleza de esta afición, que se engloba en un ámbito privado, de alto nivel adquisitivo y de una edad avanzada (Libro Blanco Federación de Golf de Madrid, 2013). No cabe duda de que, dado el perfil de los usuarios de las redes sociales (INE, 2017), estas ofrecen al sector una oportunidad para darse a conocer y divulgar este deporte entre aficionados más jóvenes. En relación a este punto se observa que, en 2017, el 67,6\% de los usuarios de Internet en los últimos tres meses participaron en redes sociales como Facebook, Twitter o YouTube; sin embargo, son los jóvenes de 16 a 24 años los que alcanzan índices de participación más elevados (90\%) (INE, 2017).

Aunque recientemente Twitter ha duplicado el número de caracteres permitido, hasta entonces el límite se establecía en 140 caracteres, siguiendo a Boyd Golder y Lotan (2010), la limitación de espacio de Twitter facilita la comunicación rápida y efectiva, así como el debate e intercambio de información, pero también requiere una comunicación regular con contenidos relevantes y específicos. Según diversos autores, Twitter se caracteriza por ser una red orientada a la atención al cliente que facilita la creación de relaciones individuales entre la empresa y el usuario (Meso-Ayerdi, Mendiguren-Galdospin y Pérez-Dasilva, 2015; Pérez-DaSilva et al., 2013). Según IAB (2017), son este tipo de plataformas las que obtienen más publicaciones. Sin embargo, también requieren un mayor esfuerzo por parte de la compañía, que está obligada a responder los mensajes con relativa celeridad. 
En consecuencia, el propósito de este trabajo es analizar cómo las empresas de campos de golf de la Comunidad de Madrid usan el servicio de Twitter para la comunicación con sus públicos, examinando el grado de efectividad de las prácticas relativas a los contenidos y aquellas orientadas a fomentar la interactividad.

\section{Metodología}

Con el objetivo de identificar las cuentas que los clubs con campos de golf mantienen activas en Twitter, se realizó un primer seguimiento en esta red social de los 37 clubs identificados por la Federación de Golf. De las 20 cuentas identificadas en Twitter, una de ellas fue desechada debido a que corresponde a un club con otros deportes sobre los que versaban la mayoría de los tuits. De estos, se seleccionaron aquellas cuentas que rondaban los 100 tuits o más a diecisiete de febrero del 2017 y desde que se creó el perfil. No obstante, también se incluyó una cuenta que solo contaba con noventa tuits, pero que su reciente creación muestra una gestión activa.

De los nueve perfiles incluidos en el análisis, dos corresponden a empresas de naturaleza pública (ostentan esta titularidad y no limitan el acceso, previo pago), dos tienen titularidad privada (su propiedad corresponde a los socios y su uso es privativo de estos) y cinco tienen un carácter comercial (es un club privado pero se permite jugar a otros jugadores no socios previo pago).

En una segunda fase, se aplicó un análisis de contenido a las cuentas corporativas que los clubs de golf de la Comunidad de Madrid mantienen activas en la red de Twitter. El análisis se extendió temporalmente a lo largo del año 2016: desde el 1 de enero hasta el 31 de diciembre. Los contenidos han sido analizados con SPSS v.22.

Tabla 1.- Número de tuits, retuits, mensajes retuiteados y "me gusta" registrados entre 1 de enero y el 31 de diciembre de 2016 en las cuentas activas de Twitter de los clubs de golf de la Comunidad de Madrid

\begin{tabular}{|l|c|c|c|c|c|c|}
\hline Cuenta & Tuits & Retuits & Retuiteado & Me gusta & Foto & Vídeo \\
\hline Encíngolf & 303 & 154 & 250 & 255 & 204 & 8 \\
\hline @elrobledalgolf & 132 & 54 & 93 & 92 & 106 & 1 \\
\hline @golfoliva & 45 & 8 & 15 & 27 & 41 & 2 \\
\hline @ctg-golf-madrid & 72 & 2 & 43 & 13 & 17 & 0 \\
\hline @cngolfmadrid & 287 & 70 & 169 & 213 & 226 & 6 \\
\hline @golfjardinaran & 175 & 26 & 76 & 83 & 43 & 2 \\
\hline @retamaresgolf & 100 & 64 & 86 & 76 & 65 & 6 \\
\hline @rcglaherreria & 95 & 59 & 87 & 87 & 69 & 5 \\
\hline @la penuela & 401 & 278 & 344 & 358 & 315 & 45 \\
\hline
\end{tabular}

Fuente. Elaboración propia

\subsection{Variables examinadas}

1. Número de tuits.

2. Retuits: mensajes que se remiten de otras cuentas para difundirlos entre sus propios seguidores. 
3. Multimedia: inclusión de fotos y/o videos.

4. Temática de los tuits publicados, no excluyentes, cuyo sistema de categorización fue:

- Autopromoción: Información de productos y servicios ofrecidos, cursos, campeonatos o productos de las tiendas.

- Información sobre eventos propios: horarios de salida, clasificaciones, resultados,...

- Logros de sus usuarios, profesionales del centro: (ejemplo: no hace mucho entrenaba aquí, hoy es un grande de..., enhorabuena).

- Patrocinios del campo, Acción social y Responsabilidad social corporativa.

- Información sobre acontecimientos y noticias del mundo del golf no vinculados a la empresa: campeonatos, torneos, premios, rankings, eventos especiales, personalidades vinculadas al golf.

- Educativo: consejos, recomendaciones, demostraciones, nuevos materiales, tutoriales...

- Estilo de vida: actividades de ocio relacionadas no vinculadas a la actividad de la empresa: noticias sobre salud, gastronomía, otros deportes, viajes....

- Saludos estacionales o diarios.

- Preguntas, concursos, sorteos, que facilitan la interacción.

- Enlaces patrocinados.

5. Me gusta, antes llamado Favorito. Permite guardar tuits en un apartado de nuestra propia cuenta para volver a leerlos cuando uno quiera.

6. Retuiteado: mensajes que los seguidores de las cuentas postean para compartir con sus propios seguidores, contribuyendo a difundir los mensajes de las cuentas analizadas.

7. № de Comentarios seguidores, categorizados según:

- Petición de información/pregunta.

- Mensajes positivos en relación a la marca, apoyo emocional y autoexpresión: incluye risas o emoticonos.

- Mensajes neutros que aportan información adicional.

- Mensajes negativos: Quejas y críticas respecto a la empresa.

- Comentarios no relacionados con la marca.

Para garantizar la fiabilidad de la medición, los dos investigadores que codificaron los contenidos de los perfiles aplicaron el esquema de las categorías propuestas a una muestra temporal de las unidades de análisis determinada al azar. En este primer test, se identificaron las áreas de confusión y se aclararon las dudas surgidas respecto a la asignación de las unidades de registro a las diversas categorías, fijando el marco de la codificación final.

\section{Resultados}

\subsection{Interactividad en la cuentas según la temática de los mensajes}

En este apartado se analiza la difusión de los tuits de los clubs de golf en otras cuentas (retuiteado), la selección de "me gusta" y las respuestas de los usuarios en relación al ámbito temático de los tuits publicados. Los datos examinados incluyen: el total de mensajes retuiteados, la proporción de tuits publicados que son retuiteados al menos en una ocasión, la media de retuits obtenidos por cada mensaje publicado, el número máximo de retuits conseguido por un tuit y la desviación típica o dispersión en el número de ocasiones que un mensaje ha sido retuiteado y, por tanto, difundido en otras cuentas. 
En primer lugar, señalar que el $72,2 \%$ de los 1610 mensajes publicados han sido retuiteados en alguna ocasión por los seguidores, y el $75 \%$ ha conseguido uno o más "me gusta". La totalidad de los tuits que aportan consejos, recomendaciones, demostraciones, nuevos materiales o tutoriales, y que hemos etiquetado como educativos, han conseguido ser difundidos o valorados como favoritos y, además, son los que han conseguido hacerlo en mayor número de ocasiones: cada tuit educativo ha sido difundido 34,2 veces y ha sido marcado con "me gusta" en 17,3 ocasiones como media (tablas 1 y 2 ). Sin embargo, su volumen total es bastante limitado y la variabilidad en su difusión y la selección de "me gusta" conseguidos es muy amplia.

Tabla 2.- Totales, porcentaje respecto al total de tuits emitidos, media, máximo y desviación típica de mensajes retuiteados por otros usuarios distribuidos por temáticas

\begin{tabular}{|l|c|c|c|c|c|}
\hline \multicolumn{1}{|c|}{ Temáticas } & Totales & $\%$ & Media & Máximo & SD \\
\hline Autopromoción & 419 & 69,37 & 1,8 & 19 & 2,3 \\
\hline Información eventos propios & 274 & 59,69 & 1,8 & 28 & 2,8 \\
\hline Logros propios usuarios/profesionales & 59 & 84,29 & 2,9 & 34 & 4,3 \\
\hline Patrocinios, acción social y RSC & 7 & 77,78 & 1,4 & 5 & 1,5 \\
\hline Noticias golf & 129 & 81,13 & 5,5 & 240 & 19,4 \\
\hline Educativo & 6 & 100,00 & 34,2 & 159 & 62,6 \\
\hline Estilo de vida & 40 & 93,02 & 4,7 & 17 & 3,2 \\
\hline Saludos & 217 & 88,57 & 4,7 & 18 & 3,7 \\
\hline Preguntas, concursos, sorteos, $\ldots$ & 2 & 66,67 & 8,7 & 24 & 13,3 \\
\hline Enlaces patrocinados & 10 & 83,33 & 3,3 & 11 & 3,5 \\
\hline
\end{tabular}

Fuente. Elaboración propia

Con un mayor número de publicaciones, el $93 \%$ de los mensajes de estilo de vida referentes a actividades de ocio no vinculadas con la empresa han sido retuiteados, y el $97,7 \%$ se han valorado con un "me gusta". Sin embargo, obtienen una media de difusión y "me gusta" mucho menor que preguntas-concursos-sorteos y noticias de golf.

Los saludos, que en buena medida son retuits de mensajes de otras cuentas por parte de los gestores de los perfiles analizados, también se posicionan en un buen lugar, superando en la media de "me gusta" obtenidos por cada tuit a las publicaciones de estilo de vida. Por el contrario, patrocinios, acción social y RSC, la información sobre eventos propios y autopromoción se sitúan en los últimos lugares en cuanto a la media de difusión y señalados como favoritos por los usuarios. 
Tabla 3.- Totales, porcentaje respecto al total de tuits emitidos, media, máximo y desviación típica de "me gusta" distribuidos por temáticas

\begin{tabular}{|l|c|c|c|c|c|}
\hline \multicolumn{1}{|c|}{ Temáticas } & Totales & $\%$ & Media & Máximo & SD \\
\hline Autopromoción & 431 & 71,36 & 2,4 & 32 & 3,2 \\
\hline Información eventos propios & 282 & 61,44 & 2,5 & 37 & 4,1 \\
\hline Logros propios usuarios/profesionales & 63 & 90,00 & 6 & 180 & 21,4 \\
\hline Patrocinios, acción social y RSC & 8 & 88,89 & 1,6 & 4 & 1,2 \\
\hline Noticias golf & 127 & 79,87 & 8,4 & 544 & 44 \\
\hline Educativo & 6 & 100,00 & 17,3 & 63 & 23,6 \\
\hline Estilo de vida & 42 & 97,67 & 8,3 & 28 & 5,1 \\
\hline Saludos & 231 & 94,29 & 9,4 & 39 & 6,7 \\
\hline Preguntas, concursos, sorteos,... & 2 & 66,67 & 10,7 & 30 & 16,8 \\
\hline Enlaces patrocinados & 12 & 100,00 & 4,9 & 16 & 4,6 \\
\hline
\end{tabular}

Fuente. Elaboración propia

De los 1610 tuits publicados en las cuentas de los clubs con campos de golf, solo 175 han recibido comentarios de los seguidores: un $11 \%$. De nuevo son los mensajes educativos y las preguntas-concursos-sorteos los que en mayor porcentaje obtienen respuestas de los usuarios, con una media por tuit más elevada. Estos datos contrastan con las publicaciones de patrocinios, acción social y RSC que no han obtenido ningún comentario, aunque es necesario precisar que esta constituye la temática menos mencionada en los tuits. No ocurría así con los mensajes de autopromoción que, siendo los mensajes más comúnmente publicados, apenas un $5 \%$ obtienen comentarios entre los seguidores.

Tabla 4.- Totales, porcentajes respecto al total de tuits emitidos, media, máximo y desviación típica de comentarios de los seguidores distribuidos por temáticas

\begin{tabular}{|l|c|c|c|c|c|}
\hline \multicolumn{1}{|c|}{ Temáticas } & Totales & $\%$ & Media & Máx & SD \\
\hline Autopromoción & 30 & 4,97 & 0,08 & 4 & 0,42 \\
\hline Información eventos propios & 53 & 11,55 & 0,28 & 16 & 1,24 \\
\hline Logros propios usuarios/profesionales & 7 & 10,00 & 0,13 & 2 & 0,41 \\
\hline Patrocinios, acción social y RSC & 0 & 0,00 & 0 & 0 & 0 \\
\hline Noticias golf & 26 & 16,35 & 0,64 & 11 & 2,05 \\
\hline Educativo & 2 & 33,33 & 1,17 & 4 & 1,83 \\
\hline Estilo de vida & 8 & 18,60 & 0,21 & 2 & 0,52 \\
\hline Saludos & 48 & 19,59 & 0,28 & 9 & 0,79 \\
\hline Preguntas, concursos, sorteos,... & 1 & 33,33 & 1 & 3 & 1,73 \\
\hline Enlaces patrocinados & 0 & 0,00 & 0 & 0 & 0 \\
\hline
\end{tabular}

Fuente. Elaboración propia 
De los 383 comentarios realizados por los usuarios, la mayoría son peticiones de información en relación a información sobre: eventos propios -129 solicitudes de información referentes a 87 mensajes publicados de esta temática-; y noticias sobre el golf -102 sobre 75 . Aunque hay que destacar que dos de seis tuits sobre contenidos educativos han generado más peticiones de información.

También son frecuentes los mensajes positivos en respuesta a saludos. Cabe resaltar que, a pesar de las críticas a las redes sociales como espacios canalizadores de quejas y comentarios negativos, estos resultan testimoniales en el caso de los perfiles en Twitter de los clubs de golf.

Tabla 5.- Tipo de comentarios de los seguidores según temática. En totales.

\begin{tabular}{|l|c|c|c|c|c|c|c|}
\hline \multicolumn{1}{|c|}{ Temáticas } & $\begin{array}{c}\text { Petición } \\
\text { de } \\
\text { información }\end{array}$ & $\begin{array}{c}\text { Queja/ } \\
\text { sugerencia }\end{array}$ & $\begin{array}{c}\text { Aporta } \\
\text { información }\end{array}$ & $\begin{array}{c}\text { Mensaje } \\
\text { positivo }\end{array}$ & $\begin{array}{c}\text { Mensaje } \\
\text { neutro }\end{array}$ & $\begin{array}{c}\text { Quejas/ } \\
\text { críticas }\end{array}$ & $\begin{array}{c}\text { Comentarios } \\
\text { ajenos a la } \\
\text { empresa }\end{array}$ \\
\hline Autopromoción & 24 & 0 & 2 & 19 & 4 & 3 & 1 \\
\hline $\begin{array}{l}\text { Información } \\
\text { eventos propios }\end{array}$ & 87 & 2 & 1 & 23 & 11 & 0 & 5 \\
\hline $\begin{array}{l}\text { Logros } \\
\text { usuarios/ } \\
\text { profesionales }\end{array}$ & 0 & 0 & 0 & 9 & 0 & 0 & 0 \\
\hline $\begin{array}{l}\text { Patrocinios, } \\
\text { acción social y } \\
\text { RSC }\end{array}$ & 0 & 0 & 0 & 0 & 0 & 0 & 0 \\
\hline Noticias golf & 75 & 1 & 1 & 19 & 5 & 0 & 1 \\
\hline Educativo & 2 & 3 & 1 & 0 & 1 & 1 & 0 \\
\hline Estilo de vida & 2 & 0 & 0 & 8 & 0 & 0 & 0 \\
\hline Saludos & 1 & 0 & 0 & 64 & 2 & 0 & 1 \\
\hline $\begin{array}{l}\text { Preguntas, } \\
\text { concursos, } \\
\text { sorteos,... }\end{array}$ & 0 & 0 & 1 & 0 & 0 & 3 & 0 \\
\hline $\begin{array}{l}\text { Enlaces } \\
\text { patrocinados }\end{array}$ & 0 & 0 & 0 & 0 & 0 & 0 & 0 \\
\hline Totales & 191 & 6 & 6 & 142 & 23 & 7 & 8 \\
\hline
\end{tabular}

Fuente. Elaboración propia

\subsection{Interactividad de las cuentas en relación con el uso de recursos multimedia y retuits}

A continuación se examina la difusión de los tuits de los clubs de golf en otras cuentas, la selección de "me gusta" y las respuestas de los usuarios a los mensajes publicados en relación a la inclusión recursos audiovisuales o si estos han sido retuiteados desde otras cuentas.

En cuanto al uso de recursos multimedia, se observa que la incorporación de fotos aumenta la probabilidad de ser retuiteado o de ser marcado como "me gusta". No sucede así con la probabilidad de recibir comentarios por parte de los seguidores, que apenas se modifica. 
Por otra parte, la incorporación de videos a los tuits incrementa sus opciones de ser difundido, obtener "me gusta" o ser comentado, aún en mayor medida que las fotos. No obstante, este es un recurso escasamente utilizado: únicamente se han contabilizado 75 tuits con videos frente a 1086 que contienen fotos, o lo que es lo mismo, el 67,5\% de los tuits incorporan fotos en tanto que solo el $4,7 \%$ hace lo propio con los videos.

Tabla 5.- Difusión, "me gusta" y comentarios de los mensajes obtenidos como media por cada tuit según sus características

\begin{tabular}{|l|c|c|c|}
\hline & $\begin{array}{c}\text { Difusión } \\
\text { (Media) }\end{array}$ & $\begin{array}{c}\text { "Me gusta" } \\
\text { (Media) }\end{array}$ & Comentarios (Media) \\
\hline Mensaje no retuiteado & 1,1 & 1,8 & 0,12 \\
\hline Mensaje retuiteado & 5,1 & 7,9 & 0,37 \\
\hline No contiene foto & 2,1 & 3 & 0,23 \\
\hline Con foto & 3,2 & 5,2 & 0,24 \\
\hline No contiene video & 2,7 & 4,1 & 0,22 \\
\hline Con video & 6,3 & 13 & 0,47 \\
\hline
\end{tabular}

Fuente. Elaboración propia

Para finalizar, la probabilidad de obtener "likes", ser difundido o comentado por los seguidores es muy superior entre los mensajes retuiteados desde otras cuentas, con porcentajes más elevados que los recursos multimedia ya analizados. En este caso, el recurso al retuit es bastante común, con $44,4 \%$ de todos los mensajes publicados por los gestores de cuentas que provienen de otras cuentas ( 715 retuits de 1610 tuits).

\section{Discusión}

Este trabajo investiga la eficacia de la gestión que los clubs con campos de golf de la Comunidad de Madrid han seguido en sus cuentas de Twitter a lo largo del año 2016, considerando la respuesta obtenida por parte de los seguidores. En base a los resultados de estudios anteriores que vinculan la actitud hacia las compañías con el grado de interactividad en redes sociales, se analiza la difusión de los tuits de clubs de golf en otras cuentas, la selección de "me gusta" y las respuestas de los usuarios a los mensajes publicados en relación a la temática tratada, la inclusión o no de recursos audiovisuales y contenidos retuiteados de otras cuentas.

La primera conclusión del estudio es que los mensajes que incorporan contenidos educativos, como consejos, recomendaciones, demostraciones, nuevos materiales o tutoriales, obtienen una respuesta muy positiva por parte de los seguidores de las cuentas en Twitter de las empresas de campos de golf. Asimismo, las publicaciones de estilo de vida que hacen referencia a otras actividades de ocio complementarias del golf, como noticias sobre salud, gastronomía, otros deportes o viajes, destacan por obtener unos buenos índices de interactividad. Muy similares son los resultados de los tuits de saludos, que alcanzan un porcentaje elevado de comentarios positivos de los seguidores, al igual que noticias de golf.

Los resultados anteriores son coherentes con las expectativas de Sung y Kim (2014), quienes valoran que los mensajes orientados a generar una relación cercana con los usuarios a través 
de conversaciones casuales y que incluyen consejos útiles sobre la vida, hablan de temas actuales o incluyen saludos estacionales o diarios generan una mayor interactividad y una actitud más positiva hacia la organización. Sin embargo, al contrario que en nuestro trabajo, los resultados empíricos de su estudio no validaron que la inclusión de mensajes no promocionales generara una percepción más favorable hacia las empresas en Facebook que las que incluyen mensajes promocionales o mixtos. Una posible explicación de estas diferencias puede vincularse con la técnica de investigación utilizada, que en el caso del estudio referido se basaba en un experimento con encuesta online, o con las características de la empresa concreta que estos autores analizaron: Walmart.

Por otra parte, se aconseja seleccionar mejor las preguntas, concursos, sorteos, que obtienen una buena media de interacción, pero concentran la difusión y preferencia en dos tercios de los mensajes, y los comentarios en un tercio de ellos, además de alguna crítica.

Los mensajes de autopromoción y corporativos (información sobre eventos propios o patrocinios, acción social y RSC) obtienen una respuesta muy pobre en cuanto al grado de interacción alcanzado entre los usuarios. Estos resultados coinciden con los registrados en el estudio de Etter (2013), quien encuentra un bajo nivel de interactividad entre los mensajes de responsabilidad social corporativa.

Otro aspecto destacable hace referencia a la frecuencia de mensajes que generan comentarios positivos en el conjunto total de intervenciones de los seguidores, y en particular los que se generan a partir de los mensajes que contienen saludos. Este dato contrasta con la limitada presencia de quejas y comentarios negativos que suele asociarse con la plataforma de Twitter. Sin duda, este resultado constituye un aliciente para los gestores de las cuentas de los clubs con campos de golf.

Para finalizar, cabe mencionar la respuesta positiva que obtienen los contenidos retuiteados y los videos en la forma de comentarios, marcados como favoritos o su difusión por los seguidores a otras cuentas. Aspecto que contrasta con el escaso uso que se hace de estos últimos, y que es aconsejado por autores como Men y Tsai (2012), Kim, Kim y Sung (2011) y Gao (2016). Igualmente, los expertos recomiendan hacer retuits (Herrera y Requejo, 2012) para aumentar el compromiso de los seguidores.

\section{Conclusiones}

Podemos concluir que Twitter constituye una plataforma apropiada para que los clubs con campos de golf involucren a un público más joven. Sin embargo, es necesaria una gestión adecuada de sus contenidos para lograr el compromiso de los usuarios. En particular, este estudio incide en la necesidad de atender a las temáticas que se abordan en las redes sociales $y$, en el caso concreto de las empresas de campos de golf, encuentra que las más populares son aquellas que incluyen consejos, recomendaciones, demostraciones, nuevos materiales o tutoriales. También obtienen niveles más elevados de respuesta los tuits que recogen noticias sobre salud, gastronomía, otros deportes, viajes y, en general, actividades de ocio no vinculadas directamente con el club. La inclusión de concursos, preguntas o sorteos es una formula conveniente para conseguir la participación de los usuarios, pero se precisa una selección adecuada de los mismos. 
Algunos de los consejos que se derivan de estos resultados apuntan a la inclusión de contenidos relevantes, entretenidos y útiles para los seguidores, sin limitar el uso de Twitter como una herramienta orientada exclusivamente al autobombo y la promoción del club y sus productos. Así mismo, se recomienda compartir contenidos de otros perfiles y saludos para generar una relación cercana con los usuarios. Como punto final, se ha observado la efectividad de la inclusión de videos, por encima de las fotos, para fomentar la interactividad de los seguidores.

Por último, aunque la información extraída proporciona resultados relevantes en cuanto a la interactividad que genera el uso de diferentes prácticas en la gestión de las cuentas de los campos de golf en Twitter, debemos considerar las limitaciones de la muestra que contiene un escaso número de unidades de análisis. Por otra parte, esta investigación se circunscribe a un sector muy concreto, el de los campos de golf, por lo que resultaría conveniente replicar esta investigación en otras esferas del deporte y de las organizaciones, en general. A este respecto, sería interesante comparar si la actividad de los seguidores en respuesta a los contenidos publicados y el uso de diversos recursos varían en función de si se trata de una empresa pública o privada.

\section{Referencias bibliográficas}

[1]Aced, C. y Lalueza, F. (2016). “¿Qué contenido publican las empresas en los medios sociales? Análisis crítico del discurso de las compañías del IBEX 35 y del Fortune 500 en blogs corporativos, Facebook y Twitter". Revista internacional de Relaciones Públicas, 11 (6), 135-154.

[2]Aymerich, F. y Anabitarte, J. (2016). El impacto económico del golf en España. Golf Business partners. Últ. Consulta 27 dic. 2017 [En línea] https://goo.gl/9WkAW2

[3] Boyd, d., Golder, S. y Lotan, G. (2010). "Tweet, Tweet, Retweet: Conversational Aspects of Retweeting on Twitter". HICCS-43. IEEE: Kauai, H1, Hanuary 6. Últ. consulta, 28 dic. 2017 [En línea] https://www.danah.org/papers/TweetTweetRetweet.pdf

[4]Bowen, S. A. (2013). "Using classic social media cases to distill ethical guidelines for digital engagement". Journal of Mass Media Ethics, 28 (2), 119-133. Últ. consulta, 28 dic. 2017 [En línea] http://www.deirdrebreakenridge.com/wp-content/uploads/2013/07/JMME Bowen 2013 SM Ethics.pdf

[5]Cornelissen, J. (2008). Corporate Communication. A guide to Theory and Practice. London: SAGE Publications.

[6]Dijkmans, C., Kerkhof, P., Buyukcan-Tetik, A. y Beukeboom, C.J. (2015). "Online Conversation and Corporate Reputation: A Two-Wave Longitudinal Study on the Effects of Exposure to the Social Media Activities of a Highly Interactive Company". Journal of Computer-Mediated Communication, 20 (6), 632-648.

[7]Edman, H. (2010). Twittering to the Top: A Content Analysis of Corporate Tweets to Measure Organization-Public Relationships. (Master's thesis), Louisiana State University, EEUU. Últ. consulta, 28 dic. 2017 [En línea] http://etd.Isu.edu/docs/available/etd-04292010-162453/unrestricted/edmanthesis.pdf

[8]Etter, M. (2013). "Reasons for low levels of interactivity (Non-) interactive CSR communication in twitter". Public Relations Review, 39, 606-608

[9]Farranha, A. C., y dos Santos, L.T. (2015). "Administração pública, direito e redes sociais: o caso da CGU no Facebook". Revista Eletrônica do Curso de Direito da UFSM, 10 (2), 742-767.

[10]Federación de Golf de Madrid (2017). Evolución de licencias. Últ. consulta, 29 dic. 2017 [En línea] http://www.fedgolfmadrid.com/ Federación de Golf de Madrid (2013). Libro blanco de 
la Federación de Golf de Madrid. Federación de Golf de Madrid.

[11]Gao, F. (2016). "Social Media as a Communication Strategy: Content Analysis of Top Nonprofit Foundations' Micro-blogs in China". International Journal of Strategic Communication, 10 (4), 255-271.

[12]Grunig, J.E. (2011). "Public relations and strategic management: Insititutionalizing organization-public relationships in contemporary society, Central European Journal of Communication". 1, 11-31. Últ. consulta, 28 dic. 2017 [En línea] http://cejsh.icm.edu.pl/cejsh/element/ bwmeta1.element.desklight-69a525a7-ea71-4b47-981d-31fa059ad02a

[13]Hallahan, K. (2006). Online public relations. En H. Bidgoli (Ed.), Handbook of information security, 3, (769-783). Hoboken, NJ: John Wiley.

[14]Herrera, S. y Requejo, J.L. (2012). "10 Good Practices form News Organizations using Twitter". Journal of Applied Journalism and Media Studies, 1 (1), 79-95.

[15] Interactive Advertising Bureau (IAB) (2016a). IV Estudio de la actividad de las marcas en medios sociales. Últ. consulta, 28 dic. 2017 [En línea] http://www.iabspain.net/wp-content/ uploads/downloads/2016/02/Estudio-de-la-Actividad-de-las-Marcas-en-MMSS vReducida.pdf (15/05/2017).

[16]IAB (2016b). Estudio Anual de Redes Sociales 2017. Últ. consulta, 28 dic. 2017 [En línea] http://iabspain.es/wp-content/uploads/iab estudioredessociales 2017 vreducida.pdf

[17]IAB (2017). IV Estudio anual de redes sociales. Últ. consulta, 28 dic. 2017 [En línea] http:// iabspain.es/wp-content/uploads/iab estudioredessociales 2017 vreducida.pdf

[18]Instituto Nacional de Estadística (INE) (2017). Encuesta sobre equipamiento y uso de tecnologías de información y comunicación en los hogares. Año 2016. Últ. consulta, 26 dic. 2017 [En línea]. http:// www.ine.es

[19]Kang, M. (2014). "Understanding public engagement: Conceptualizing and measuring its influence on supportive behavioral intentions". Journal of Public Relations Research, 26, 399416. Últ. consulta, 29 dic. 2017 [En línea] http://dx.doi.org/10.1080/1062726X.2014.959863

[20]Kim, E., Sung, Y. y Kang, H. (2014). "Brand followers' retweeting behavior on Twitter: How brand relationships influence brand electronic word-of-mouth". Computers in Human Behavior, 37, 18-25.

[21]Kim, S., Kim, S. Y. y Sung, K. H. (2011). How Fortune 100 companies are employing corporate communication strategies on Facebook: Corporate ability versus corporate social responsibility. Presentation at the International Communication Association Conference, May 2011, Boston, MA.

[22]Li, Z. y Li, C. (2014). "Tweet or "re-tweet"? An experiment of message strategy and interactivity on Twitter". Internet Research, 24 (5), 648-667.

[23]McCorkindale, T. (2010). "Can you see the writing on my wall? A content analysis of the Fortune 50's Facebook social networking sites". Public Relations Journal, 4 (3), 1-14. Últ. consulta, 26 dic. 2017 [En línea] http://citeseerx.ist.psu.edu/viewdoc/download?doi=10.11.470.6602\&rep=rep1\&type $=$ pdf

[24]Men, L.R. y Tsai, W.S. (2015). "Infusing social media with humanity: Corporate character, public engagement and relational outcomes". Public Relations Review, 41, 395-403.

[25]Men, L.R., y Tsai, W.S (2013). "Beyond liking or following: Understanding public engagement on social networking sites in China". Public Relations Review, 39 (19), 13:22

[26]Men, L.R. Y Tsai, W.S. (2012). "How companies cultivate relationships with publics on social network sites: Evidence from China and the United States". Public Relations Review, 38, 723-730. 
[27]Meso-Ayerdi, K., Mendiguren-Galdospin, T. y Pérez-Dasilva, J.A. (2015). "The Role of Community Manager in the Marketing Strategies of the Spanish Companies Most Active on Social Networks". Estudios sobre el Mensaje Periodístico, 21 (1), 385-402

[28]Moreno, A., Molleda, J.C., Athaydes, A. y Suárez, A.M. (2017). Latin American Communication Monitor 2016-2017. Tendencias en comunicación estratégica: big data, automatización, engagement, influencers, couching y competencias. Resultados de una encuesta en 17 países. Madrid, España: DIRCOM/EUPRERA. Últ. consulta, 28 dic. 2017 [En línea] http:// latincommunicationmonitor.com/site/wp-content/uploads/2017/05/LCM-2016-2017.pdf

[29]Pérez-Dasilva, J.A., Genaut-Arratibel, A., Meso-Ayerdi, K., Mendiguren-Galdospín, T., Marauri-Castillo, I., Iturregui-Mardaras, L., Rodríguez-González, MM. y Rivero-Santamarina, D. (2013). "Las empresas en Facebook y Twitter. Situación actual y estrategias comunicativas". Revista Latina de Comunicación Social, 68, 676-695. Últ. consulta, 29 dic. 2017 [En línea] http://www.revistalatinacs.org/068/paper/996 Bilbao/RLCS paper996.pdf

[30]Ruiz-Iniesta, C. (2012). "El uso de las herramientas digitales por parte de los bancos. El caso de la imagen en Internet de Banco Santander y BBVA en época de crisis". Revista Internacional de Relaciones Públicas, 4 (II), 51-72. Últ. consulta, 28 dic. 2017 [En línea] http:// revistarelacionespublicas.uma.es/index.php/revrrpp/article/view/118/82

[31]Sisson, D.C. (2017). "Control mutuality, social media, and organization-public relationships: A study of local animal welfare organizations' donors". Public Relations Review, 43, 179-189.

[32]Sung, K.H. y Kim, S. (2014). "I Want to Be Your Friend: The Effects of Organizations' Interpersonal Approaches on Social Networking Sites". Journal of Public Relations Research, 26, 235-255.

[33]Vernuccio, M. (2014). "Communicating Corporate Brands Through Social Media: An Exploratory Study". International Journal of Business Communication, 51(3), 211-233.

[34]Voss, K.a. y Kumar, A. (2013). "The value of social media: are universities successfully engaging their audience?" Journal of Applied Research in Higher Education, 5 (2), 156-172.

[35]Watkins, B.A. (2017). "Experimenting with dialogue on Twitter: An examination of the influence of the dialogic principles on engagement, interaction, andattitude". Public Relations Review. 43, 163-171.

[36]Wright, DK. y Hinson, MD. (2015). "Examining Social and Emerging Media Use in Public Relations Practice: A Ten-Year Longitudinal Analysis". Public Relations Journal, 9 (2). Últ. consulta, 26 dic. 2017 [En línea] http://prjournal.instituteforpr.org/wp-content/uploads/2015v09n02WrightHinson.pdf 


\section{CURRICULUM VITAE}

\section{María Cruz López de Ayala}

Licenciada en Sociología (UCM), doctora en Ciencias de la Comunicación (URJC) y máster en Dirección de Comunicación por la Universidad de Barcelona. Profesora del área de Publicidad y Comunicación Audiovisual en la Universidad Rey Juan Carlos. Sus líneas de investigación son medios de comunicación y menores, la comunicación política local y Comunicación Corporativa. Ha participado en proyectos de investigación competitivos $(I+D+I)$ y publica en revistas nacionales e internacionales de impacto. Es ponente regular en Congresos nacionales e internacionales.

\section{Beatriz Catalina García}

Licenciada en Periodismo por la UCM (1989), doctora en Ciencias de la Comunicación por la Universidad Rey Juan Carlos (2011) y licenciada en Ciencias Políticas (UNED, 2014). Profesora visitante del área de Periodismo de la Universidad Rey Juan Carlos, sus trabajos publicados están relacionados con la comunicación política local y los usos de Internet. Ha participado proyectos competitivos $\mathrm{I}+\mathrm{D}+\mathrm{I}$ y ha publicado en numerosas revistas nacionales e internacionales de impacto. También participa regularmente con ponencias en congresos nacionales e internacionales.

\section{José Gabriel Fernández}

Profesor en la URJC. Licenciado en Ciencias de la Información por la Universidad de Navarra,doctor en Ciencias de la Información (UCM) y máster en Periodismo Económico (UCM). Autor de diversas publicaciones sobre periodismo especializado en economía y deporte, ha participado en diferentes congresos internacionales sobre estas especialidades. Ha sido redactor de Diario 16 y del canal 24 Horas de TVE. Colabora habitualmente en madridiario.es y en Radio Marca, y ha colaborado en Radio Intereconomía, entre otros. 